\title{
Profile of total IgG, IgG1, IgG2, IgG3 and IgG4 levels in sera of patients with paracoccidioidomycosis: treatment follow-up using Mexo and rPb27 as antigens in an ELISA
}

\author{
Lílian da Silva Santos ${ }^{1 /+}$, Viviane Cristina Fernandes ${ }^{3}$, Samuel Gonçalves da Cruz², \\ Weverton César Siqueira², Alfredo Miranda Goes ${ }^{3}$, Ênio Roberto Pietra Pedroso ${ }^{1,2}$ \\ 1Programa de Pós-Graduação em Ciências da Saúde, Infectologia e Medicina Tropical ${ }^{2}$ Faculdade de Medicina ${ }^{3}$ Departamento de \\ Bioquímica e Imunologia, Instituto de Ciências Biológicas, Universidade Federal de Minas Gerais, Belo Horizonte, MG, Brasil
}

The levels of total of $\operatorname{Ig} G, \operatorname{IgG1}, \operatorname{IgG2}, \operatorname{Ig} G 3$ and $\operatorname{IgG4}$ were evaluated in 54 patients with chronic paracoccidioidomycosis (PCM) before, during and after treatment using an enzyme-linked immunosorbent assay with Mexo and recombinant Pb27 (rPb27) as the antigens. Mexo was effective in distinguishing PCM patients from individuals in the negative control group (NC) based on total IgG and rPb27 performed worse than Mexo when these two groups were compared. IgG1, IgG2, IgG3 and IgG4 could not be used to clearly distinguish PCM patients from those in the NC group using either antigen. There was no clear relationship between antibody levels and the period of treatment. The majority of patients presented with decreased antibody levels during treatment, with no statistically significant differences among the different periods of treatment. Only IgG4 presented a negative correlation between its levels and clinical improvement during treatment. In total, $65 \%$ of untreated PCM patients showed reactivity against IgG4 when the Mexo antigen was used and this reactivity decreased over the course of treatment. There was a tendency towards decreasing antibody levels during treatment, but these antibody levels did not necessarily clear after the treatment was stopped. Mexo was useful for PCM diagnosis using total IgG; however, more studies are necessary before this antigen can be used in measuring the levels of total IgG and its subclasses for monitoring patients during treatment.

Key words: treatment follow-up - ELISA - Mexo antigen - rPb27 antigen - paracoccidioidomycosis - IgG subclasses

Paracoccidioidomycosis (PCM) is a systemic disease caused by the thermodimorphic fungus Paracoccidioides brasiliensis (Marques 1998). This deep mycosis is endemic in many Latin American countries, with the majority of cases occurring in Brazil, followed by Venezuela, Colombia, Ecuador and Argentina (ShikanaiYasuda 2006, Ameen et al. 2010). The most affected age group is between $30-50$ years old, $90 \%$ of whom are men who live in rural areas and work in agriculture. Recently, endemic foci of PCM infection were found in urban areas, which can be related to population migration from rural to urban areas (Blotta et al. 1999). Epidemiological studies have demonstrated that the number of patients clinically diagnosed with PCM may represent only a small proportion of infected individuals (Almeida et al. 2003). In endemic areas, up to $50 \%$ of inhabitants have been exposed to the fungus, but only a minority develops the disease (Ameen et al. 2010).

PCM is characterised as an infection when patients are positively diagnosed using serological, microbiological or molecular techniques, even in the absence of signs

Financial support: CAPES, FAPEMIG, CNPq

+ Corresponding author: lilianufop@yahoo.com.br

Received 16 December 2010

Accepted 25 November 2011 or symptoms of the disease because many patients can be asymptomatic at the time of first evaluation. The evolution of PCM infection can resolve spontaneously, progress to disease or remain latent, depending on the immune response of the host (Rivitti \& Aoki 1999). PCM disease may manifest with several symptoms ranging from local and benign to disseminated, severe and progressive, leading to fatal outcomes in the absence of treatment. The clinical manifestations of PCM disease may vary depending on several factors, such as the virulence of the fungus, the host's established immune response, the affected areas and other intrinsic factors of the host (Benard 2008, Mendes-Giannini et al. 2008). There are two clinical forms of PCM disease: the acute, or juvenile form, and the adult, or chronic form (Franco et al. 1987, Ramos-e-Silva et al. 2008). The acute form affects children and adolescents of both genders and represents $5-15 \%$ of all cases. This clinical form is characterised by a rapid and aggressive progression, mainly affecting the reticuloendothelial system. Frequent manifestations include skin lesions, digestive symptoms and lymphadenopathy (Nogueira et al. 2006). The chronic form occurs in more than $90 \%$ of patients, most of them adult males between $30-50$ years old. This form progresses slowly, with pulmonary symptoms present in $90 \%$ of the affected adults (Shikanai-Yasuda et al. 2006, Wanke \& Aidê 2009).

PCM is associated with a decrease in the cellular immune response and an increase in the humoral immune response. Patients with severe forms of PCM have strong 
polyclonal B cell activation, hypergammaglobulinaemia and high levels of specific antibodies, which are generally correlated with disease severity (Del Negro et al. 2000, Juvenale et al. 2001, Shikanai-Yasuda et al. 2006).

The assessment of the humoral immune response is an important tool for the diagnosis and follow-up care of PCM patients. Different serological techniques are used to measure the levels of IgG, such as double immunodiffusion (DI), counterimmuno-electrophoresis (CIE), immunofluorescence (IFI), enzyme-linked immunosorbent assay (ELISA) and immunoblotting. These techniques may use various antigenic preparations to assess antibodies against $P$. brasiliensis. Some of these techniques use crude antigenic preparations, while others use specific antigenic preparations, such as the $19 \mathrm{kDa}, 31 \mathrm{kDa}$, $43 \mathrm{kDa}$ and $70 \mathrm{kDa}$ glycoproteins and recombinant $\mathrm{Pb} 27$ $(\mathrm{rPb} 27)$ (Ortiz et al. 1998, Baida et al. 1999, Díez et al. 2003, Albuquerque et al. 2005, Correa et al. 2007, Reis et al. 2008, Fernandes et al. 2011, Silveira-Gomes et al. 2011). However, there is no consensus on the best techniques for the diagnosis and follow-up care of PCM patients (Campos et al. 1990, Alves 1996, Martins et al. 1997, Del Negro et al. 2000, Camargo 2008).

Different techniques have been employed to measure the levels of $\mathrm{IgG}$ and its subclasses IgG1, IgG2, IgG3 and IgG4. Some groups have attempted to associate classes of immunoglobulins with clinical forms of PCM or clinical improvement during treatment (Mota \& Franco 1979, Barbosa et al. 1981, Biagioni et al. 1984, Baida et al. 1999, Del Negro et al. 2000, Juvenale et al. 2001). However, the relationship between the levels of immunoglobulin subclasses and clinical improvement remains controversial.

Different classes of drugs can be used for the treatment of PCM, including sulphonamides (sulphamethoxazoletrimethoprim), amphotericin $\mathrm{B}$, imidazole derivatives (ketoconazole, itraconazole and fluconazole) and triazolic derivatives (voriconazole). Drug selection is based on disease severity, but the treatment cost can be an important factor in drug choice (Shikanai-Yasuda et al. 2006).

This study aimed to measure the serum levels of total IgG, IgG1, IgG2, IgG3 and IgG4 in both untreated PCM patients and patients after different treatment durations via an ELISA with two different antigenic preparations (Mexo and $\mathrm{rPb} 27$ ) to verify the suitability of these antigens for use in the diagnosis and follow-up care of PCM patients.

\section{SUBJECTS, MATERIALS AND METHODS}

Patients and control sera - Sera were collected from 54 patients with chronic PCM before, during and after treatment at the Training Center and Parasitic Infectious Diseases Reference, Hospital and Clinics (HC) of Federal University of Minas Gerais (UFMG), Brazil. A total of 92 serum samples were assessed and, of these, 38 were obtained from the same patients at different time points during and after treatment. Sera were aliquoted and stored at $-20^{\circ} \mathrm{C}$ until use. The diagnosis of PCM was determined by biopsy in all patients and, in some cases, conventional serological tests were used in combination with the biopsy results. Patients were treated with ketoconazole, itraconazole, sulphamethoxazole-trimeth- oprim or amphotericin B during hospitalisation. The patients in this study were not treated with immunosuppressive drugs. The first analysis evaluated one serum sample from each PCM patient before or at the beginning of treatment to compare the levels of total $\mathrm{IgG}$ and its subclasses with those found in healthy individuals (NC group) to show the suitability of Mexo and $\mathrm{rPb} 27$ as antigens for use in PCM diagnosis. This assay was performed on the 54 initial serum samples from the PCM patients and 10 serum samples from the NC group. Next, a second analysis was performed to assess the levels of total IgG and its subclasses during PCM treatment. To accomplish this analysis, a total of 92 serum samples were obtained (many patients provided more than one serum sample during treatment) and the results of these samples were compared with those from the NC group. Serum samples were classified according to the duration of time over which the patients were treated, the time elapsed since the end of treatment as follows: not treated (NT) (14), treated for one month (T1M) (8), treated for two-nine months (T2-9M) (19), treated for one year (T1Y) (13), treated for two years (T2Y) (11), treated for three-four years (T3-4Y) (16), six-nine months from the end of treatment (AT6-9M) (3), one year from the end of treatment (AT1Y) (2), two years from the end of treatment (AT2Y) (2) and three years from the end of treatment (ATY3) (1). Additionally, one group contained patients who had relapsed (Rel) (3). In both experiments, sera from $10 \mathrm{NC}$ from the Institute of Biological Sciences (UFMG) were assessed to determine cut-off values of the ELISA. Patients with concomitant diseases, such as toxoplasmosis, histoplasmosis, cryptococcosis, infectious mononucleosis, acquired immune deficiency syndrome, tuberculosis, sarcoidosis or lymphoma, were excluded from the study. This study was approved by the Ethical Committee of the HC of School of Medicine of UFMG and informed consent was obtained from each patient before blood collection.

Antigens - The secreted and surface antigen Mexo was obtained from Pb18, a human source of a virulent strain of P. brasiliensis (Reis et al. 2005). Yeast cells were cultured in YPD agar medium $(0.5 \%$ yeast extract, $0.5 \%$ peptone, $1.5 \%$ D-glucose and $1.5 \%$ agar, $\mathrm{pH} 7.0$ ) (Sigma, USA) at $35^{\circ} \mathrm{C}$ and harvested on the seventh day of culture. Yeast cells were removed from the culture medium and subjected to agitation by a vortex in 0.05 mol L-1 phosphate buffered saline (PBS), $\mathrm{pH} 7.4$, for $30 \mathrm{~s}$. The solution was centrifuged $(14,000 \mathrm{~g})$ for $10 \mathrm{~min}$ at $4^{\circ} \mathrm{C}$. The amount of protein in the supernatant was quantified using the Bradford method (Bradford 1976) and this preparation was used as the Mexo antigen.

The $\mathrm{rPb} 27$ antigen was obtained as follows. The sequence of the recombinant $\mathrm{rPb} 27$ had already been cloned by our group into the expression vector pGEX 4T-2 (Gibco BRL) (GST), as described by Reis et al. (2008). To facilitate the purification procedure, the $\mathrm{rPb} 27$ sequence was transferred, according to the manufacturer's instructions, into the expression vector pETDEST 42 (Invitrogen, Carlsbad, USA), which allowed for the expression of the recombinant protein with a 
C-terminal his-tag. The protein $\mathrm{rPb} 27$ was expressed in Escherichia coli which according to the manufacturer's instructions produces a recombinant protein with a Cterminal his-tag. Purification of the recombinant protein was performed using a HiTrap ${ }^{\mathrm{TM}}$ Chelating HP (Amersham Biosciences, Uppsala, Sweden).

ELISA of total $\operatorname{IgG}, \operatorname{IgG1}, \operatorname{IgG2}, \operatorname{IgG3}$ and IgG4 levels using the Mexo and rPb27 antigens - The ELISAs of anti- $P$. brasiliensis total IgG, IgG1, IgG2, IgG3 and IgG4 levels were performed in flat-bottomed polystyrene plates (Nunc-ImmunoPlate PolySorp Surface, USA) using the Mexo and $\mathrm{rPb} 27$ antigens. Briefly, plates were coated overnight at $4^{\circ} \mathrm{C}$ with $100 \mu \mathrm{L}$ of a $1 \mu \mathrm{g} / 100 \mu \mathrm{L}$ solution of Mexo or $\mathrm{rPb} 27$ in a $0.5 \mathrm{~mol} \mathrm{~L}^{-1}$ carbonate buffer, $\mathrm{pH}$ 9.6. The plates were washed five times with washing buffer $\left[0.05 \mathrm{~mol} \mathrm{~L}^{-1} \mathrm{PBS}\right.$ with $0.05 \%$ Tween 20 (PBS-Tween)] and blocked with $200 \mu \mathrm{L}$ of blocking solution [1.5 mol L-1 PBS with $1.6 \%$ casein (PBS-casein)] for $1 \mathrm{~h}$ at $37^{\circ} \mathrm{C}$. After incubation, the plates were washed five times with PBS-Tween and filled with $100 \mu \mathrm{L}$ of either patient sera or negative control sera (in duplicate) diluted $1: 400$ in $1.5 \mathrm{~mol} \mathrm{~L}^{-1} \mathrm{PBS}$ with $0.25 \%$ casein. The plates were re-incubated for $1 \mathrm{~h}$ at $37^{\circ} \mathrm{C}$ and then washed 10 times. After washing, $100 \mu \mathrm{L}$ of a rabbit anti-human total $\mathrm{IgG}$ peroxidase-conjugated antibody specific to the gamma chain (DAKO, USA) diluted 1:10.000 in 0.15 mol L-1 PBS was added to the wells. This antibody reacts specifically with the gamma chain, detecting only $\operatorname{IgG}$ antibodies. The plates were incubated for $1 \mathrm{~h}$ at $37^{\circ} \mathrm{C}$ and then washed 10 times. The reaction was developed with $100 \mu \mathrm{L}$ of TMB Plus (Bio-tecnologia, Brazil) for $10 \mathrm{~min}$ at room temperature. Colour development was stopped with $50 \mu \mathrm{L}$ of $2 \mathrm{~mol} \mathrm{~L}^{-1} \mathrm{H}_{2} \mathrm{SO}_{4}$. The optical density (OD) at $450 \mathrm{~nm}$ was determined using an ELISA reader (Anthos 2010, Cambridge, England). Similar protocols were performed for the IgG subclasses, but because these monoclonal antibodies were not conjugated, a goat anti-mouse peroxidase-conjugated antibody was added as an additional step. The monoclonal anti-human IgG1 antibody (Fc-specific, Sigma, USA) was used at a 1:12.000 dilution and a goat anti-mouse IgG2b peroxidase-conjugated antibody $(\gamma 2 \mathrm{~b}$-chain specific, SouthernBiotech, USA) was added at a 1:6.000 dilution. The monoclonal anti-human IgG2, IgG3 and IgG4 antibodies (Sigma, USA) were used at a 1:10.000 dilution and a goat anti-mouse IgG1 peroxidase-conjugated antibody $(\gamma 1-$ chain-specific, SouthernBiotech) was added at a 1:6.000 dilution. Cut-off values for the detection of IgG and its subclasses were determined using the mean plus three standard deviations of serum levels from $10 \mathrm{NC}$.

Statistical analysis - Serological results of the PCM patients vs. the negative control group (NC) were analysed using the Mann-Whitney test. The groups of patients treated for different durations of time, NT and the NC groups were compared and analysed using the KruskallWallis non-parametric test. The comparison between all groups was calculated using Dunn's test. The Spearman rank correlation coefficient was used in correlation studies. All data were considered significant when $\mathrm{p}<0.05$.

\section{RESULTS}

In this study, the sera from 54 patients with chronic PCM were assessed before, during and after treatment. A total of 92 serum samples were analysed using an inhouse ELISA with two different antigenic preparations (Mexo and $\mathrm{rPb} 27$ ) to determine total IgG, IgG1, IgG2, $\mathrm{IgG} 3$ and $\mathrm{IgG} 4$ levels. First, one serum sample from each patient (before treatment or at the beginning of treatment) was analysed and compared with NC group. Significant differences were found between serum samples from the PCM group (54 patients with PCM) and the NC group (sera from $10 \mathrm{NC}$ ) using Mexo as the antigen for total IgG and its subclasses. The in-house ELISA using Mexo as the antigen showed higher antibody reactivity for total IgG in all PCM sera analysed. IgG2, IgG1, IgG4 and $\mathrm{IgG} 3$ showed decreasing antibody reactivity, respectively. Only one PCM patient had an OD value below the cut-off point when total IgG was measured. In contrast, for IgG1, IgG2, IgG3 and IgG4, many PCM patients had OD values below the cut-off point (Fig. 1). When the $\mathrm{rPb} 27$ antigen was used, statistically significant differences were found between the PCM patients and the NC group with respect to total $\mathrm{IgG}, \mathrm{IgG} 1, \mathrm{IgG} 2$ and $\mathrm{IgG} 4$. Using $\mathrm{rPb} 27$, total IgG also showed higher antibody reactivity, which was similar to the results observed for the Mexo antigen. However, of the IgG subclasses, IgG1 had the highest antibody reactivity, followed by IgG4, IgG2 and IgG3, respectively. Using $\mathrm{rPb} 27,10$ patients had OD values below the cut-off point for total IgG. For IgG1, $\operatorname{IgG} 2, \operatorname{IgG} 3$ and $\operatorname{IgG} 4$, the majority of patients did not have OD values above the cut-off point (Fig. 2).

When the 92 serum samples from the different treatment conditions of the 54 PCM patients were analysed, according to the duration of treatment or time since the end of treatment, statistically significant differences were observed among the different groups of patients for total $\mathrm{IgG}, \mathrm{IgG} 1$ and $\mathrm{IgG} 2$ using Mexo and $\mathrm{rPb} 27$. IgG4 showed statistically significant differences only with the Mexo antigen. No significant differences were observed for IgG3 using either the Mexo antigen $(p=0.3548)$ or the $\mathrm{rPb} 27$ antigen $(\mathrm{p}=0.2711)$. The analysis of total $\operatorname{IgG}$ levels using the Mexo antigen showed statistically significant differences $(\mathrm{p}<0.05)$ when the NT, T1M, T2-9M, T1Y and Rel groups were compared with the NC group (Fig. 3). When the $\mathrm{rPb} 27$ antigen was used, statistically significant differences were observed when the NT, T29M, T1Y and Rel groups were compared with the NC group (Fig. 4). Statistically significant differences were observed for IgG1 using the Mexo antigen when the NT, T1M, T2-9M, T1Y and Rel groups were compared with the NC group (Fig. 3). When $\mathrm{rPb} 27$ was used, a statistically significant difference was only observed between the Rel group and the NC group (Fig. 4). IgG2 analysis using the Mexo antigen showed statistically significant differences when among T2-9M and Rel groups were compared with the NC group (Fig. 3). Using the rPb27 antigen, only the T2-9M group was significantly different from the NC group (Fig. 4). IgG4 analysis using Mexo as the antigen showed a statistically significant difference when the NT and T2-9M groups were compared to the $\mathrm{NC}$ group. Only this analysis showed a negative correla- 


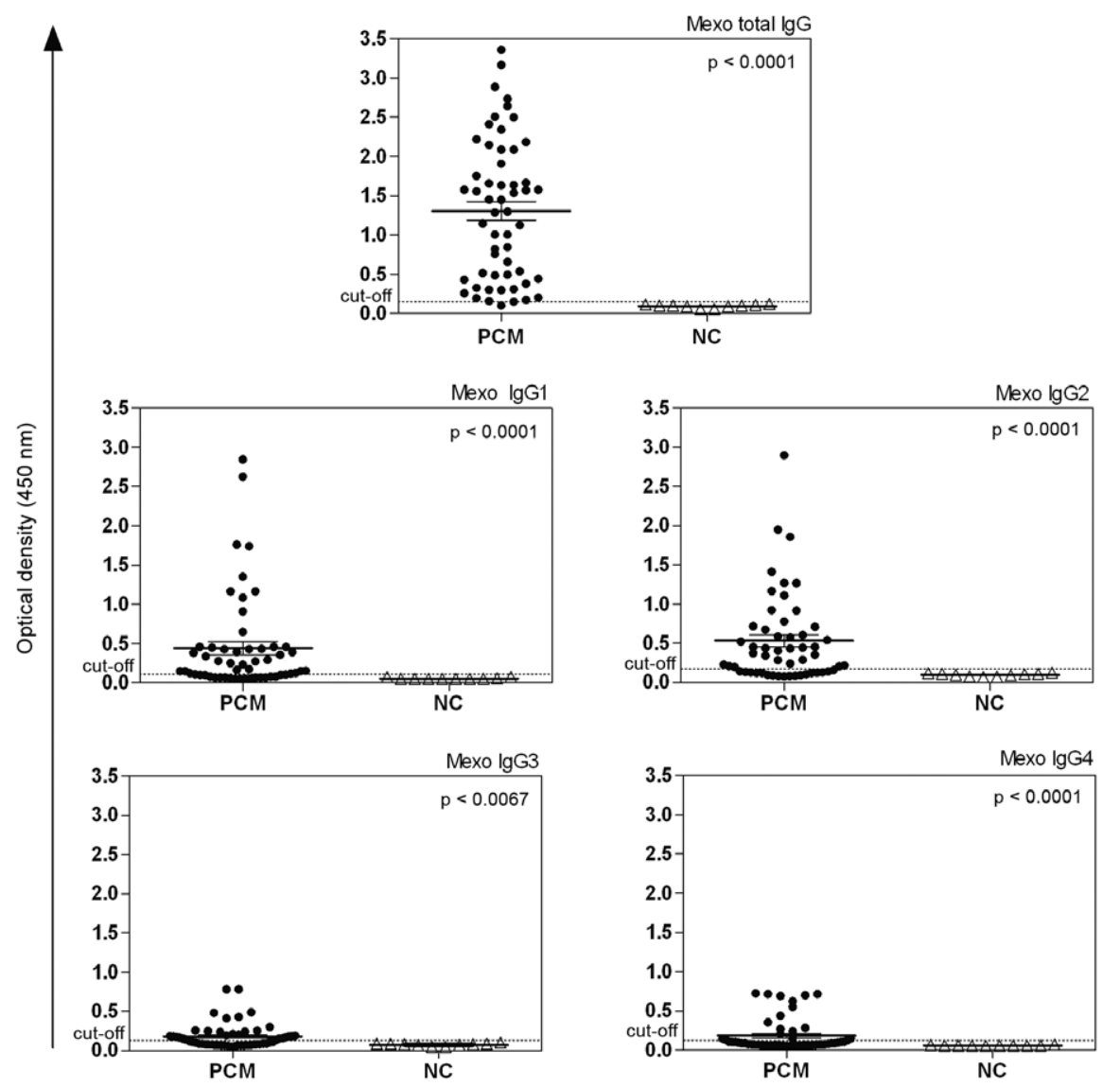

Fig. 1: total IgG, IgG1, IgG2, IgG3 and IgG4 serum levels measured by an in house enzyme-linked immunosorbent assay technique using Mexo antigen in the sera of 54 patients with chronic paracoccidioidomycosis (PCM) and 10 subjects from negative control group (NC). Each dot represents the optical density of a single patient and the horizontal lines represent the median value of the group. The cut-off point is represented by the dotted line. The statistical significant level is indicated for each group ( $\mathrm{p}$ value).

tion between the treatment period and $\mathrm{OD}$ of the IgG4 serum. Among sera from NT patients, $65 \%$ showed reactivity with IgG4 and this reactivity clearly decreased as the treatment progressed (Fig. 3). When $\mathrm{rPb} 27$ was used, no statistically significant differences were found $(\mathrm{p}=$ 0.3014) (Fig. 4). It is worth mentioning that all groups were compared with each other, but statistically significant differences were only found between the NC group and the NT group or between the NC group and other groups with different periods of treatment as explained above. No significant difference was observed among the different treated groups.

Patients were also analysed according to their anti-fungal therapy and their clinical disease states. Of 54 patients, only four did not undergo treatment during this study. The remaining 50 patients were divided as follows: 38 were treated with sulphamethoxazole-trimethoprim, seven with itraconazole and five with ketoconazole. The analysis of patients treated with sulphamethoxazole-trimethoprim did not reveal any association between IgG levels and the duration of treatment. Some patients with more than one year of treatment had $\operatorname{IgG}$ values similar to patients at the beginning of treatment. For the other subclasses of $\mathrm{IgG}$ assayed using Mexo and $\mathrm{rPb} 27$, patients had values similar to the cut-off point. All patients treated with ketoconazole had three years of treatment and two of them had high levels of total IgG, measured using Mexo in one case and $\mathrm{rPb} 27$ in the other. Patients treated with itraconazole had between one month and nine months of treatment and of seven patients, five had high levels of total $\operatorname{IgG}$ using Mexo and $\mathrm{rPb} 27$, regardless of the duration of treatment. No patients were treated exclusively with amphotericin B. This drug was prescribed to a few patients during hospitalisation and it was always combined with itraconazole, ketoconazole or sulphamethoxazole-trimethoprim. For this reason, it was not possible to verify the effect of amphotericin B on antibody levels. Supplementary data gives the medians and standard deviations of the levels of total IgG and its subclasses in untreated patients and those treated with sulphamethoxazole-trimethoprim, ketoconazole or itraconazole. Supplementary data shows the similarities in the levels of $\mathrm{IgG}$ and its subclasses between the different treatment groups.

We also tested for an association between clinical status and antibody level, but no correlation was found. Of 54 patients, 23 presented with localised lesions and were classified as having unifocal disease, and 31 patients who presented with lesions in more than one area of the body were 


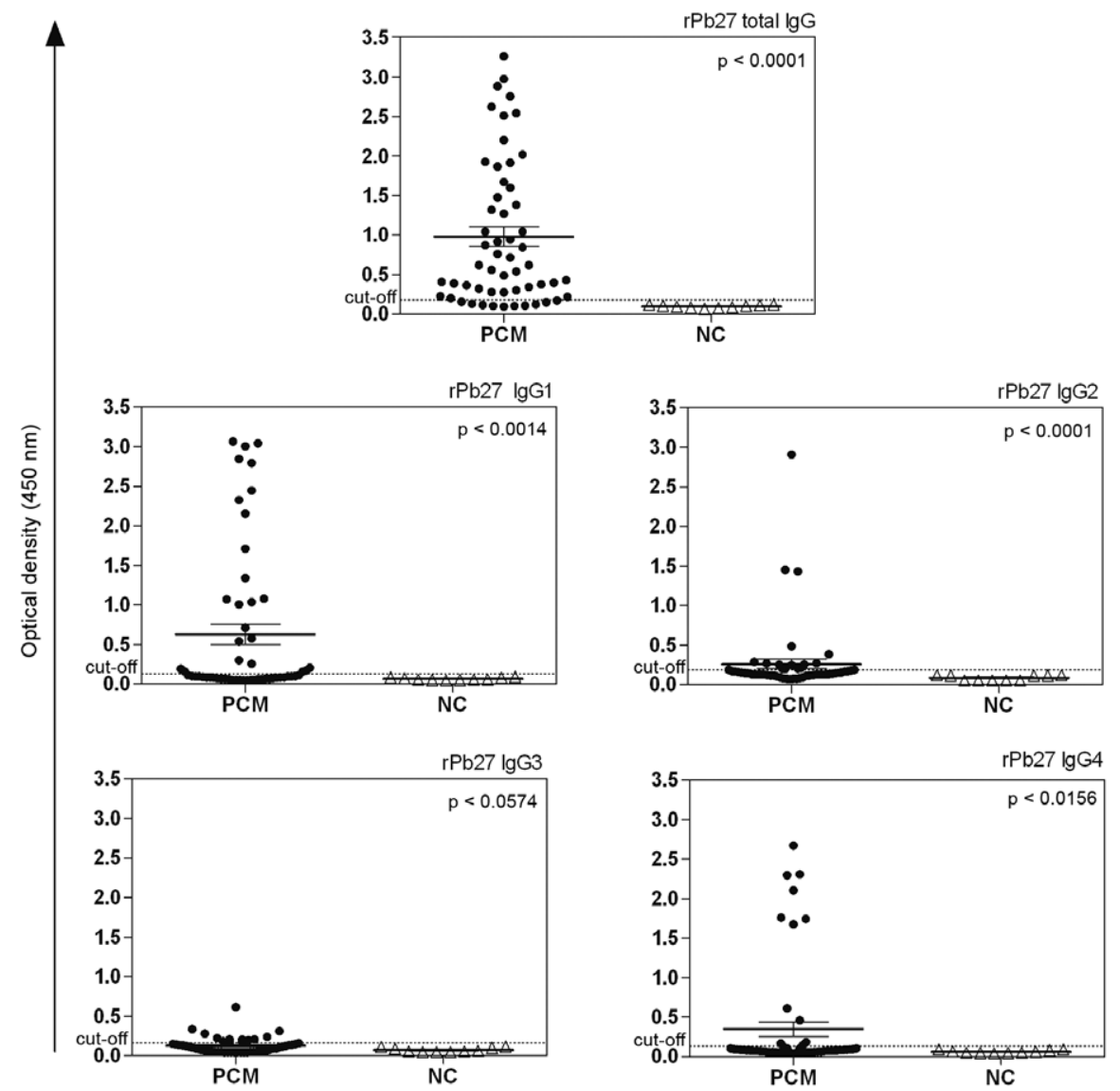

Fig. 2: total IgG, IgG1, IgG2, IgG3 and IgG4 serum levels measured by an in house enzyme-linked immunosorbent assay technique using recombinant $\mathrm{Pb} 27(\mathrm{rPb} 27)$ antigen preparation in the sera of 54 patients with chronic paracoccidioidomycosis $(\mathrm{PCM})$ and 10 subjects from negative control group $(\mathrm{NC})$. Each dot represents the optical density of a single patient and the horizontal lines represent the median value of the group. The cut-off point is represented by the dotted line. The statistical significant level is indicated for each group ( $\mathrm{p}$ value).

classified as having multifocal disease. Similar levels of IgG and its subclasses were detected in patients with unifocal and multifocal diseases, with no statistically significant differences for almost all antibodies. Only the analysis of $\mathrm{IgG} 2$ using $\mathrm{rPb} 27$ as the antigen yielded a statistically significant difference between the unifocal and multifocal groups. However, this difference was related to only two patients from the unifocal group that presented with high levels of IgG2, and there is no clear explanation for this result. Supplementary data shows the medians and standard deviations of the levels of total IgG and its subclasses in patients in relation to their clinical disease status.

\section{DISCUSSION}

In this study, 92 serum samples from PCM patients at different points in treatment or after treatment were analysed. All of the patients selected had chronic PCM, varying from unifocal to multifocal disease. The first aim of our study was to assess the utility of Mexo and $\mathrm{rPb} 27$ as antigens using an ELISA. This evaluation showed statistically significant differences between PCM serum samples and the NC group for total $\mathrm{IgG}$ and all of its subclasses when Mexo was used as the antigen. Using Mexo, total IgG showed the highest level of reactivity followed by IgG2, IgG1, IgG4 and IgG3, respectively. The Mexo antigen has already been shown to have high sensitivity and specificity for the detection of antibodies against $P$. brasiliensis. Reis et al. (2005) analysed the serological responses of $140 \mathrm{PCM}$ patients to the Mexo antigen using immunoblotting, ELISA and IFI techniques. They observed a homogenous $\operatorname{IgG}$ response against the Mexo antigen with high sensitivity and specificity. In our study, we confirmed the value of Mexo as an antigen to discriminate between infected and non-infected serum samples using total $\operatorname{IgG}$ analysis. Only one patient with PCM had OD values below the cut-off point when total IgG was assessed, which shows the suitability of this antigen for use in PCM diagnosis. Previous studies have shown that some patients with PCM may not be reactive to the gp43 antigen, the most-studied antigenic fraction of $P$. brasiliensis (Del Negro et al. 1991, Neves et al. 2003, Vidal et al. 2005). Many factors may be involved in this absence of a serological response to antigens in some patients, such as the prozone effect, immunosuppression or immune complex formation. However, it is not clear why the patient in our study had undetectable levels of IgG because he did not present with immunosuppression, prozone effect or immune complex formation. One 


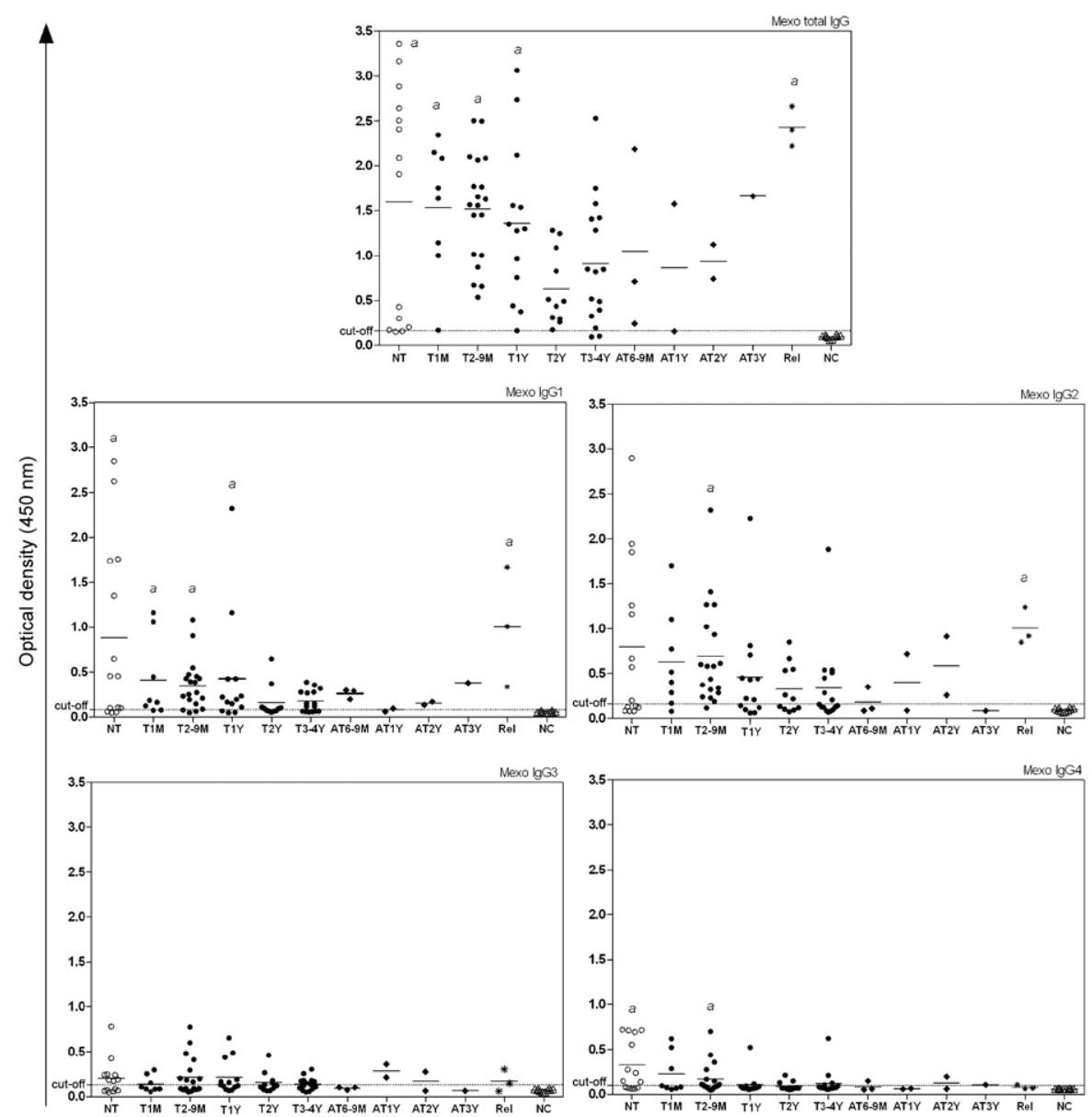

Fig. 3: total IgG, IgG1, IgG2, IgG3 and IgG4 serum levels measured by an in house enzyme-linked immunosorbent assay technique using Mexo antigen preparation in 92 sera samples of patients with chronic paracoccidioidomycosis (PCM) before treatment (NT), during treatment [treated for one month (T1M), treated for two-nine months (T2-9M), treated for one year (T1Y), treated for two years (T2Y), treated for three-four years (T3-4Y)], after treatment [one year from the end of treatment (AT1Y), two years from the end of treatment (AT2Y), three years from the end of treatment (ATY3), relapsed (Rel)] and 10 subjects from negative control group (NC). Each dot represents the optical density of a single patient and the horizontal lines represent the median value of the group. The cut-off point is represented by the dotted line. Data marked by an 'a' were significantly different $(\mathrm{p}<0.05)$ from NC group.

possible explanation for his low levels of $\operatorname{IgG}$ is that this patient presented with the unifocal form (he only had a mild oral mucosal lesion) and patients with mild forms of PCM may have a lower humoral immune response. However, many other patients with unifocal PCM had high OD values compared to multifocal PCM patients.

When $\mathrm{rPb} 27$ was used as the antigen, total $\mathrm{IgG}$ showed the highest levels of reactivity, followed by IgG1, $\mathrm{IgG} 4, \mathrm{IgG} 2$ and $\mathrm{IgG} 3$, respectively. Only the analysis of IgG3 showed no statistically significant difference between the PCM patients and the NC group. For total IgG analysis, 10 serum samples of PCM patients were observed with OD values below the cut-off point, a worse performance compared with that of the Mexo antigen. The analysis of the $\operatorname{IgG}$ subclasses did not allow us to distinguish PCM patients from the NC group. This result is understandable because unlike Mexo, $\mathrm{rPb} 27$ is a much more specific molecule, which limits the epitopes recognised by IgG. Reis et al. (2008) immunised mice with the antigenic $\mathrm{rPb} 27$ fraction and measured their humoral immunological response. These mice produced high levels of $\operatorname{IgG} 2 \mathrm{~b}$, moderate levels of IgG1 and low levels of IgG2a. The authors also observed high levels of transforming growth factor beta and interferon (IFN)- $\gamma$ and a low production of IL-10. These results suggested that $\mathrm{rPb} 27$ promoted protection against infection with $P$. brasiliensis yeast cells. It was proposed that this antigenic fraction could be used in the future as a prophylactic vaccine for PCM. The protein $\mathrm{rPb} 27$, first characterised by McEwen et al. (1996), has been used as an antigen for PCM antibody detection in several studies (Ortiz et al. 1996, 1998, Díez et al. 2003). Ortiz et al. (1996) used $\mathrm{rPb} 27$ as an antigen in immunoblotting to analyse 44 PCM patients with acute and chronic disease. The $\mathrm{rPb} 27$ antigen was recognised by antibodies from 40 PCM patients and no cross reactivity was observed with other mycoses. In a later study by this same group, $\mathrm{rPb} 27$ was used in an ELISA to detect antibodies in PCM pa- 


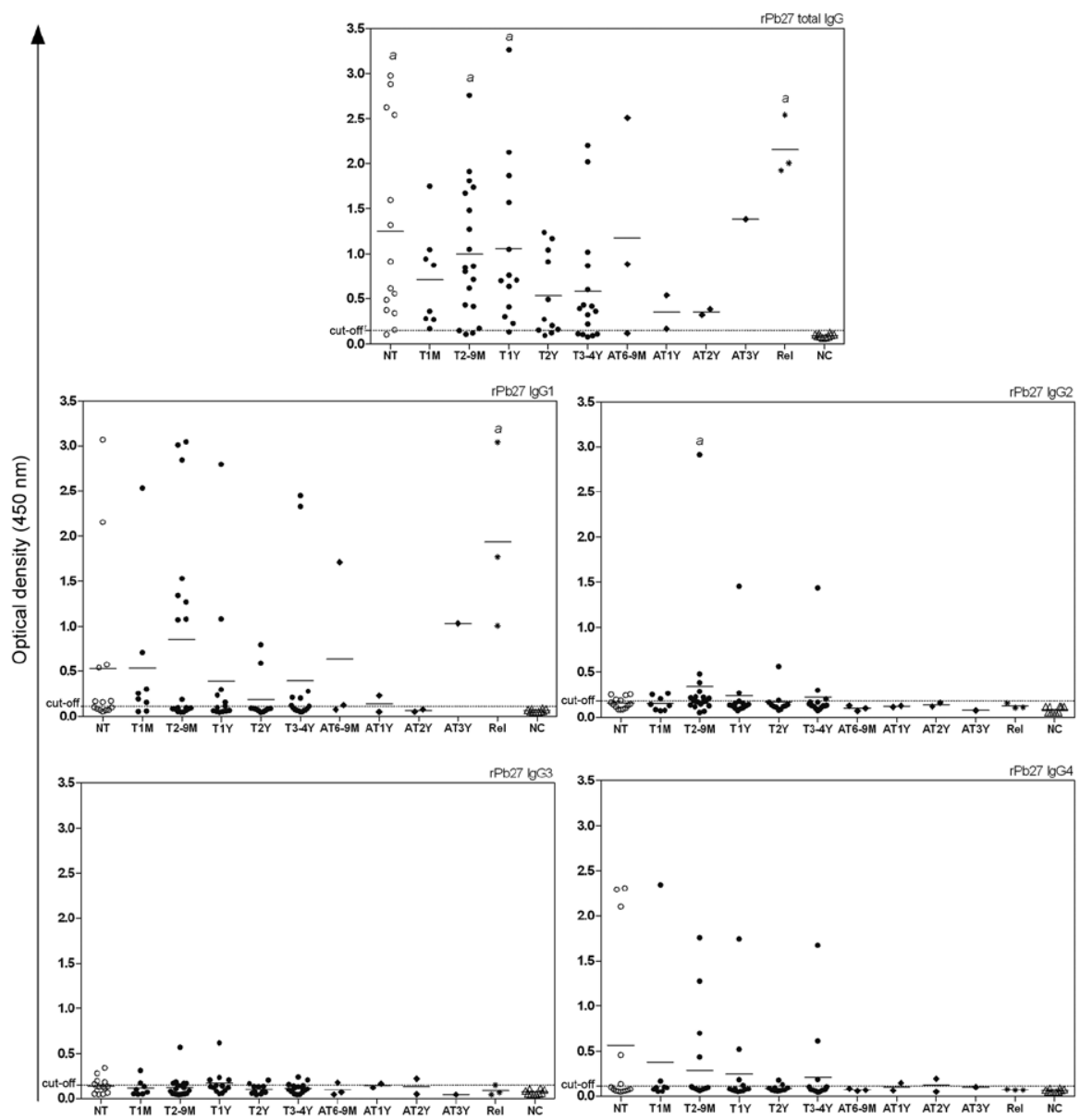

Fig. 4: total IgG, IgG1, IgG2, IgG3 and IgG4 serum levels measured by an in house enzyme-linked immunosorbent assay technique using Mexo antigen preparation in 92 sera samples of patients with chronic paracoccidioidomycosis (PCM) before treatment (NT), during treatment [treated for one month (T1M), treated for two-nine months (T2-9M), treated for one year (T1Y), treated for two years (T2Y), treated for three-four years (T3-4Y)], after treatment [one year from the end of treatment (AT1Y), two years from the end of treatment (AT2Y), three years from the end of treatment (ATY3), relapsed (Rel)] and 10 subjects from negative control group (NC). Each dot represents the optical density of a single patient and the horizontal lines represent the median value of the group. The cut-off point is represented by the dotted line. Data marked by an 'a' were significantly different $(\mathrm{p}<0.05)$ from $\mathrm{NC}$ group.

tients with different forms of clinical disease. Although the test demonstrated significant sensitivity, with antibodies from the majority of PCM patients recognising the antigen used, cross reactivity with aspergillosis and histoplasmosis serum samples was observed (Ortiz et al. 1998). Some patients not producing detectable antibodies against the $\mathrm{rPb} 27$ antigen could be related to the specificity of this protein because it has a single dominant antigenic epitope. It would likely be necessary to use a cocktail of recombinant proteins to achieve higher sensitivity values. Recently, a study performed by our group (Fernandes et al. 2011) demonstrated that the combined use of $\mathrm{rPb} 27$ and $\mathrm{rPb} 40$ in an ELISA provided high sensitivity and specificity for PCM diagnosis, which confirmed that $\mathrm{rPb} 27$ was much more efficient as an antigen when paired with another recombinant protein than when isolated.

As shown in other studies, total $\operatorname{IgG}$ is generally detected at high levels in PCM patients, especially in the acute form or in severe disease, regardless of the antigen used (Biagioni et al. 1984, Baida et al. 1999, Juvenale et al. 2001, Marquez et al. 2009). It is worth noting that all patients in this study had the chronic form of PCM and even with this form of PCM, they all presented with high levels of total $\mathrm{IgG}$ using Mexo or $\mathrm{rPb} 27$ as the antigen. However, when using IgG1 or IgG2, it was not possible to clearly distinguish PCM patients from the NC group using Mexo or $\mathrm{rPb} 27$ antigens because many PCM serum samples yielded OD values below the cut-off point. In using Mexo or rPB27 as an antigen, some studies found high levels of IgG2 in chronic PCM patients, whereas IgG1 was detected at medium levels in chronic patients. These results were confirmed in this work (Baida et al. 1999, Juvenale et al. 2001). Few serum samples of PCM patients had OD values above the cut-off value for IgG3 and IgG4 regardless of the antigen used. This result agrees with studies that observed low or absent IgG3 in PCM patients and detectable IgG4 mostly in acute PCM patients (Baida et al. 1999, Juvenale et al. 2001). 
The second part of our study determined the levels of total IgG and its subclasses in chronic PCM patients before, during and after treatment. Some groups have attempted to determine a correlation between antibody levels and disease severity in PCM patients, especially in the follow-up period after treatment (Bueno et al. 1997, Martins et al. 1997, Reis et al. 2005, Anastácio et al. 2007, Bertini et al. 2007, Yoshida et al. 2009), but this association is still controversial. Restrepo et al. (1978) found decreased antibody levels after six months of treatment using DI and CF tests in only three of the 16 patients analysed. Although apparently cured, most patients showed persistent low antibody levels. Ferreira-da-Cruz et al. (1990) used DI to measure antibodies against $P$. brasiliensis in 66 patients with PCM before and after treatment. Clinical improvement was associated with decreased antibody levels in all patients during treatment. However, after two years of treatment, nine patients continued to show high antibody levels, which showed that the measurement of IgG levels using DI does not indicate active PCM. Reis et al. (2005) demonstrated that patients with PCM undergoing treatment for more than one year had a reduced antibody response against the Mexo antigen, suggesting that the presence of antibodies against Mexo could be an indicator of active disease. In our study, untreated PCM patients had the highest OD values for total IgG when using Mexo or $\mathrm{rPb} 27$ as the antigen. However, a clear decrease in antibody levels associated with clinical improvement during treatment was not observed. Indeed, PCM patients may need several years to clear antibody levels, as suggested by Lopes (1971) and Ferreira-da-Cruz et al. (1990). Del Negro et al. (2000) evaluated antibody responses during the follow-up care of patients with acute and chronic PCM using ELISA, CIE and CF (complement fixation) techniques. In that study, 43 patients were analysed before treatment and 27 of these patients were followedup during their treatment for two years. Patients with chronic unifocal disease cleared their antibodies after one year of treatment when CF was used and after two years when ELISA was used, in contrast to the results of our study. It was suggested that patients with chronic unifocal disease needed a shorter course of therapy and patients with acute or multifocal disease needed more than two years of treatment to clear their antibodies. An increase in antibody levels was also associated with the relapse of PCM in five patients. Baida et al. (1999) assessed total IgG, IgG1, IgG2, IgG3, IgG4 and IgA in the sera of patients with juvenile and adult PCM using the $43 \mathrm{kDa}$ glycoprotein as the antigen in an ELISA. They found that juvenile PCM patients had higher total IgG levels when compared with the adult form. IgG1 levels were similar between the two groups. However, IgG4 levels were higher in the juvenile form, while IgG2 levels were higher in the adult form of PCM. In their study, IgG3 was either absent or detected at low levels in all patients, while IgA was detected mainly in the adult form of PCM. Our study also showed that for both antigens used, high total IgG levels in all patients were observed, independent of the treatment duration. Juvenale et al. (2001) found the same results in sera from acute and chronic PCM patients before treatment using a crude antigen of P. brasiliensis in an ELISA. Similar to Baida et al. (1999) and Juvenale et al. (2001), in our study, IgG3 was detected in few patients and at low levels with both antigens, independent of the treatment duration and clinical disease state. When the Mexo antigen was used, $\mathrm{IgG} 2$ was detected at high levels, especially at the baseline of treatment, but showed no differences related to clinical manifestation. Baida et al. (1999) showed higher IgG2 levels in adult PCM patients as a driven response of IFN- $\gamma$. Patients with benign or chronic PCM tend to have a Th1 immune pattern. However, patients with juvenile PCM have high IgG4 levels, suggesting a Th2 immune pattern. Because our study examined only patients with the chronic form of PCM, low levels of IgG4 in all patients could be anticipated. It is worth mentioning that a negative correlation was found only for IgG4; a decrease in IgG4 levels was associated with clinical improvement during treatment. Antibody titres tend to decrease during therapy, but not all clinically cured patients show negative serology immediately after treatment is stopped. In our study, using Mexo or $\mathrm{rPb} 27$ as the antigen, high levels of total IgG and its subclasses were detected in some patients after three years of treatment. One explanation for this result could be the severity of PCM presented by these patients. Of 54 patients, 31 presented with multifocal disease, with severe lesions in different areas of the body, including the skin, oral mucosa and lungs, simultaneously. High antibody levels may persist in patients with more severe disease for a longer period of time. In our study, patients with unifocal disease had a tendency to produce lower levels of IgG and its subclasses, but no significant difference in antibody levels was found between patients with mild and severe PCM.

The correlation between high antibody levels and relapse is also controversial. There does not seem to be a direct association between increased antibody levels and relapse in all patients. However, in our study, the three Rel patients analysed presented high levels of total $\operatorname{IgG}$ using Mexo or rPb27 as the antigen. These three Rel patients were treated with sulphamethoxazole-trimethoprim and they presented with a more severe clinical disease state, which may explain the similar levels of total $\operatorname{IgG}$ and its subclasses. The failure in the treatment with sulphamethoxazole-trimethoprim in patients with relapse is not explained by the drug alone because the majority of patients in the study were treated with this drug and they achieved clinical cure within a few months of treatment, including those with more severe clinical disease. In fact, the patients with relapse presented with a more severe clinical disease and it is possible that the drug used was not the most appropriate in an initial phase of treatment. However, many other factors may be involved in the progression of PCM disease and it was not possible to discern why these patients relapsed because they received the same care as other patients. In our study, when Mexo was used as the antigen, the three Rel patients had OD values above the cut-off point for total IgG, IgG1 and IgG2. For IgG3, only one Rel patient had an OD value above the cut-off point. When $\mathrm{rPb} 27$ was used as the antigen, patients with relapses showed an 
OD value above the cut-off point for total IgG and IgG1. This result shows the direct relationship between the measurement of antibody levels and the antigenic fractions used in the different serological tests. It is worth mentioning that the levels of total $\mathrm{IgG}$ were high in Rel patients using either Mexo or $\mathrm{rPb} 27$ as the antigen. This is an important finding because the majority of followups of PCM patients are performed for total IgG detection and not for its subclasses (Del Negro et al. 2000, Fernandes et al. 2011). However, this result does not allow for a conclusion about the relationship between relapses and increases in the levels of antibodies because only three patients presented relapses and a statistical determination could not be performed with this small group.

Ultimately, no clear association between antibody levels and treatment follow-up was found in our study. Many patients had decreased antibody levels during therapy, but there was no significant difference between the different treatment groups. A significant difference was found only between patients at different times of treatment and the NC group for total IgG and its subclasses. Only IgG4 presented a negative correlation between its levels and clinical improvement during treatment. With this result, it can be inferred that antibody levels tend to decrease during treatment, but these levels do not clear in most patients after treatment is stopped. Because many patients in this study had the multifocal form of PCM, they will likely require a longer time to clear their antibody levels. These results also suggest an increase in total $\mathrm{IgG}$ levels in patients with relapses using either Mexo or rPb27 as the antigen. Finally, the Mexo antigen was useful in discriminating between PCM patients and the NC group, especially for total $\mathrm{IgG}$, showing its suitability for use in PCM diagnosis. It is worth noting that serological follow-up of PCM patients during and after treatment is of great importance, but more studies are necessary to establish an ideal serological technique for this purpose.

\section{REFERENCES}

Albuquerque CF, Marques da Silva SH, Camargo ZP 2005. Improvement of the specificity of an enzyme-linked immunosorbent assay for diagnosis of paracoccidioidomycosis. J Clin Microbiol 43: 1944-1946.

Almeida OP, Jacks J Jr, Scully C 2003. Paracoccidioidomycosis of the mouth: an emerging deep mycosis. Crit Rev Oral Biol Med 14: $377-383$.

Alves JR 1996. Comparação entre três métodos sorológicos no seguimento de pacientes com paracoccidioidomicose, MSc Thesis, Universidade Federal de São Paulo, São Paulo, 73 pp.

Ameen M, Talhari C, Talhari S 2010. Advances in paracoccidioidomycosis. Clin Exp Dermatol 35: 576-580.

Anastácio VM, Passeto MPA, Góngora DVN, Soares MMCN, A1meida MTG 2007. Paracoccidioidomycosis: correlation between clinical and laboratorial findings in São José do Rio Preto region. Arq Cienc Saude 14: 181-185.

Baida H, Biselli PJC, Juvenale M, Del Negro GMB, Mendes-Giannini MJS, Duarte AJS, Benard G 1999. Differential antibody isotype expression to the major Paracoccidioides brasiliensis antigen in juvenile and adult paracoccidioidomycosis. Microbes Infect 1: 273-278.
Barbosa SFC, Takeda AK, Chacha J, Cuce LC, Fava Netto C 1981. Anticorpos específicos das classes IgG, IgM e IgA para Paracoccidioides brasiliensis dosados através da reação de imunofluorescência indireta no soro de pacientes e sua correlação com o tempo de evolução e forma clínica da doença. Rev Inst Adolfo Lutz 41: 121-126.

Benard G 2008. An overview of the immunopathology of human paracoccidioidomycosis. Mycopathol 165: 209-221.

Bertini S, Colombo AL, Takahashi HK, Straus AH 2007. Expression of antibodies directed to Paracoccidioides brasiliensis glycosphingolipids during the course of paracoccidioidomycosis treatment. Clin Vaccine Immunol 14: 150-156.

Biagioni LMJ, Souza MJ, Chamma LG, Mendes RP, Marques SA, Mota NGS, Franco M 1984. Serology of paracoccidioidomycosis. II. Correlation between class-specific antibodies and clinical forms of the disease. Trans R Soc Trop Med Hyg 78: 617-621.

Blotta MH, Mamoni RL, Oliveira SJ 1999. Endemic regions of paracoccidioidomycosis in Brazil: a clinical and epidemiologic study of 584 cases in the Southeast Region. Am J Trop Med Hyg 61: $390-394$

Bradford RM 1976. A rapid and sensitive method for the quantitation of microgram quantities of protein by the principle of protein-dye binding. Ann Biochem 72: 248.

Bueno JP, Mendes-Giannini MJ, Del Negro GM, Assis CM, Takiguti CK, Shikanai-Yasuda MA 1997. IgG, IgM and IgA antibody response for the diagnosis and follow-up of paracoccidioidomycosis: comparison of counterimmunoelectrophoresis and complement fixation. J Med Vet Mycol 35: 213-217.

Camargo ZP 2008. Serology of paracoccidioidomycosis. Mycopathologia 165: 289-302.

Campos EP, Unterkircher C, Camargo ZP 1990. Serological evaluation in follow-up of the paracoccidioidomycosis patients. Rev Microbiol 21: 11-17.

Correa MM, Bedoya AM, Guerrero MP, Méndez J, Restrepo A, McEwen JG 2007. Diagnosis of paracoccidioidomycosis by a dot blot assay using a recombinant Paracoccidioides brasiliensis p27 protein. Mycoses 50: 41-47.

Del Negro GM, Garcia NM, Rodríguez EG, Cano MI, de Aguiar MS, Lírio VS, Lacaz CS 1991. The sensitivity, specificity and efficiency values of some serological tests used in the diagnosis of paracoccidioidomycosis. Rev Inst Med Trop Sao Paulo 33: 277-280.

Del Negro GM, Pereira CN, Andrade HF, Palacios SA, Vidal MM, Charbel CE, Benard G 2000. Evaluation of tests for antibody response in the follow-up of patients with acute and chronic forms of paracoccidioidomycosis. J Med Microbiol 49: 37-46.

Díez S, Gómez BL, McEwen JG, Restrepo A, Hay RJ, Hamilton AJ 2003. Combined use of Paracoccidioides brasiliensis recombinant 27-kilodalton and purified 87-kilodalton antigens in an enzyme-linked immunosorbent assay for serodiagnosis of paracoccidioidomycosis. J Clin Microbiol 41: 1536-1542.

Fernandes VC, Coitinho JB, Veloso JM, Araújo SA, Pedroso EP, Góes AM 2011. Combined use of Paracoccidioides brasiliensis recombinant $\mathrm{rPb} 27$ and $\mathrm{rPb} 40$ antigens in an enzyme-linked immunosorbent assay for immunodiagnosis of paracoccidioidomycosis. J Immunol Methods 31: 78-84.

Ferreira-da-Cruz MF, Francesconi-do-Vale AC, Espinera MC, Wanke B, Galvão-Castro B 1990. Study of antibodies in paracoccidioidomycosis: follow-up of patients during and after treatment. $J$ Med Vet Mycol 28: 151-157.

Franco M, Montenegro MR, Mendes RP, Marques SA, Dillon ML, Mota NGS 1987. Paracoccidioidomycosis: a recently proposed classification of its clinical forms. Rev Soc Bras Med Trop 20: 129-132. 
Juvenale M, Del Negro GM, Duarte AJS, Benard G 2001. Antibody isotypes to Paracoccidioides brasiliensis somatic antigen in subacute and chronic form of paracoccidiodomycosis. J Med Microbiol 50: 127-134.

Lopes CF 1971. Evaluación de los resultados obtenidos en el tratamiento de la blastomicosis sudamericana con sulfamida de administración semanal. Med Cutanea 15: 357-366.

Marques AS 1998. Paracoccidioidomicose. An Bras Dermatol 73: 455-469.

Marquez ADES, Moreira AP, Leonello PC, Nakanishi FA, Itano EN 2009. Serum proteins and fractions, HDL-cholesterol and total IgG and IgE levels in cases of acute and chronic paracoccidioidomycosis. Rev Soc Bras Med Trop 42: 245-249.

Martins R, Marques S, Alves M, Fecchio D, Franco MF 1997. Serological follow-up of patients with paracoccidioidomycosis treated with itraconazole using dot-blot, ELISA and Western blot. Rev Inst Med Trop Sao Paulo 39: 187-195.

McEwen JG, Ortiz BL, García AM, Florez AM, Botero S, Restrepo A 1996. Molecular cloning, nucleotide sequencing and characterization of a $27-\mathrm{kDa}$ antigenic protein from Paracoccidioides brasiliensis. Fungal Genet Biol 20: 125-131.

Mendes-Giannini MJ, Monteiro da Silva JL, de Fátima da Silva J, Donofrio FC, Miranda ET, Andreotti PF 2008. Interactions of Paracoccidioides brasiliensis with host cells: recent advances. Mycopathologia 165: 237-248.

Mota FT, Franco M 1979. Observações sobre a pesquisa de anticorpos IgM anti-Paracoccidioides brasiliensis por imunofluorescência no soro de pacientes com paracoccidioidomicose. Rev Inst Med Trop Sao Paulo 21: 82-89.

Neves AR, Mamoni RL, Rossi CL, Camargo ZP, Blotta MSHL 2003. Negative immunodiffusion test results obtained with sera of paracoccidioidomycosis patients may be related to low-avidity immunoglobulin G2 antibodies directed against carbohydrate epitopes. Clin Diagn Lab Immunol 5: 802-807.

Nogueira MGS, Andrade GMQ, Tonelli E 2006. Clinical evolution of paracoccidioidomycosis in 38 children and teenagers. Mycopathologia 161: 73-81.

Ortiz BL, Díez S, Urán ME, Rivas JM, Romero M, Caicedo V, Restrepo A, Mcewen JG 1998. Use of the 27-Kilodalton recombinant protein from Paracoccidioides brasiliensis in serodiagnosis of paracoccidioidomycosis. Clin Diagn Lab Immunol 5: 826-830.
Ortiz BL, Garcia AM, Restrepo A, Mcewen JG 1996. Immunological characterization of a recombinant 27-kilodalton antigenic protein from Paracoccidioides brasiliensis. Clin Diagn Lab Immunol 3: 239-241.

Ramos e Silva M, Saraiva LE 2008. Paracoccidioidomycosis. Dermatol Clin 26: 257-269.

Reis BS, Bozzi A, Prado FLS, Pereira MCN, Ferreira FE, Godoy P, Moro L, Pedroso EP, Leite MF, Góes AM 2005. Membrane and extracellular antigens of Paracoccidioides brasiliensis (Mexo): identification of a $28 \mathrm{kDa}$ protein suitable for immunodiagnosis of paracoccidioidomycosis. J Immunol Methods 307: 118-126.

Reis BS, Fernandes VC, Martins EMN, Serakides R, Góes AM 2008. Protective immunity induced by $\mathrm{rPb} 27$ of Paracoccidioides brasiliensis. Vaccine 26: 5461-5469.

Restrepo A, Restrepo M, de Restrepo F, Aristizábal LH, Moncada LH, Vélez H 1978. Immune responses in paracoccidioidomycosis. A controlled study of 16 patients before and after treatment. Sabouraudia 16: 151-163.

Rivitti EA, Aoki V 1999. Deep fungal infections in tropical countries. Clin Dermatol 17: 171-190.

Shikanai-Yasuda MA, Telles Filho FQ, Mendes RP 2006. Guidelines in paracoccidioidomycosis. Rev Soc Bras Med Trop 39: 297-310.

Silveira-Gomes F, Sarmento DN, Pinto TM, Pimentel RF, Nepomuceno LB, Espírito Santo EP, Mesquita-da-Costa M, Camargo ZP, Marques-da-Silva SH 2011. Development and evaluation of a latex agglutination test for the serodiagnosis of paracoccidioidomycosis. Clin Vaccine Immunol 18: 604-608.

Vidal MSM, Benard G, Brito T, Dantas KC, Pereira CN, França FOS, Silva AMG, Martins JEC 2005. Atypical serological response marked by a lack of detectable anti-gp43 antibodies in a patient with disseminated paracoccidioidomycosis $J$ Clin Microbiol 43: 3014-3016.

Yoshida M, Sanchez MCA, Shikanai-Yasuda MA 2009. Increased immunoglobulin $\mathrm{G}$ anti-Paracoccidioides brasiliensis serum antibody avidity as a predictor of favorable post therapeutic evolution in paracoccidioidomycosis. Clin Vaccine Immunol 16: 1583-1586.

Wanke B, Aidê MA 2009. Chapter 6 - Paracoccidioidomycosis. J Bras Pneumol 35: 1245-1249. 
Levels of IgG and its subclasses measure in patients with paracoccidioidomycosis (PCM) compared according to their scheme of treatment

\begin{tabular}{|c|c|c|c|c|c|c|c|c|c|c|}
\hline \multirow{2}{*}{$\begin{array}{l}\text { Condition of } \\
\text { treatment }\end{array}$} & \multicolumn{5}{|c|}{ Mexo $($ mean \pm SD) } & \multicolumn{5}{|c|}{$\mathrm{rPb} 27($ mean $\pm \mathrm{SD})$} \\
\hline & Total IgG & IgG1 & $\operatorname{IgG} 2$ & IgG3 & IgG4 & Total IgG & $\operatorname{IgG} 1$ & IgG2 & IgG3 & IgG4 \\
\hline SMZ-TMP & $1.596 \pm 1.280$ & $0.308 \pm 0.322$ & $0.397 \pm 0.477$ & $0.175 \pm 0.131$ & $0.145 \pm 0.168$ & $0.821 \pm 0.764$ & $0.502 \pm 0.823$ & $0.291 \pm 0.491$ & $0.122 \pm 0.094$ & $0.311 \pm 0.655$ \\
\hline Ceto & $0.919 \pm 0.556$ & $0.167 \pm 0.152$ & $0.217 \pm 0.200$ & $0.106 \pm 0.044$ & $0.115 \pm 0.057$ & $0.617 \pm 0.512$ & $0.293 \pm 0.418$ & $0.151 \pm 0.089$ & $0.102 \pm 0.042$ & $0.181 \pm 0.241$ \\
\hline Itra & $0.919 \pm 0.556$ & $0.254 \pm 0.152$ & $0.725 \pm 0.523$ & $0.269 \pm 0.294$ & $0.180 \pm 0.165$ & $0.749 \pm 0.520$ & $0.328 \pm 0.302$ & $0.218 \pm 0.092$ & $0.110 \pm 0.061$ & $0.255 \pm 0.450$ \\
\hline NT & $0.919 \pm 0.556$ & $0.881 \pm 0.997$ & $0.799 \pm 0.898$ & $0.205 \pm 0.196$ & $0.323 \pm 0.284$ & $1.250 \pm 1.073$ & $0.527 \pm 0.915$ & $0.158 \pm 0.061$ & $0.141 \pm 0.087$ & $0.564 \pm 0.912$ \\
\hline
\end{tabular}

ceto: cetoconazole; itra: itraconazole; mean \pm standard deviation (SD): median of levels of IgG and its subclasses plus its SD; NT: not treated patients; $\mathrm{rPb} 27$ : recombinant Pb27; SMZ-TMP: sulphamethoxazole-trimethoprim.

Levels of IgG and its subclasses measured in patients with paracoccidioidomycosis (PCM) compared according to their clinical manifestations

\begin{tabular}{|c|c|c|c|c|c|c|c|c|c|c|}
\hline \multirow{2}{*}{$\begin{array}{l}\text { Clinical } \\
\text { form }\end{array}$} & \multicolumn{5}{|c|}{ Mexo $($ mean \pm SD) } & \multicolumn{5}{|c|}{$\mathrm{rPb} 27($ mean $\pm \mathrm{SD})$} \\
\hline & Total IgG & IgG1 & $\operatorname{IgG} 2$ & IgG3 & IgG4 & Total IgG & IgG1 & IgG2 & IgG3 & IgG4 \\
\hline Unifocal & $1.254 \pm 0.735$ & $0.364 \pm 0.576$ & $0.537 \pm 0.562$ & $0.145 \pm 0.118$ & $0.206 \pm 0.223$ & $0.821 \pm 0.734$ & $0.525 \pm 0.868$ & $0.338 \pm 0.609$ & $0.146 \pm 0.117$ & $0.271 \pm 0.613$ \\
\hline Multifocal & $1.154 \pm 0.789$ & $0.308 \pm 0.449$ & $0.361 \pm 0.412$ & $0.181 \pm 0.142$ & $0.127 \pm 0.132$ & $0.717 \pm 0.659$ & $0.398 \pm 0.612$ & $0.143 \pm 0.094$ & $0.100 \pm 0.048$ & $0.278 \pm 0.558$ \\
\hline
\end{tabular}

mean \pm standard deviation (SD): median of levels of IgG and its subclasses plus its SD; rPb27: recombinant Pb27. 